\title{
A novel DNA-binding feature of MeCP2 contributes to Rett syndrome
}

\author{
Xin Xu and Lucas Pozzo-Miller* \\ Department of Neurobiology, The University of Alabama at Birmingham, Birmingham, AL, USA \\ ${ }^{*}$ Correspondence: lucaspm@uab.edu \\ Edited by: \\ Hansen Wang, University of Toronto, Canada \\ Reviewed by: \\ Susana Cohen-Cory, University of California, USA \\ Nicoletta Landsberger, University of Insubria, Italy
}

\section{A commentary on}

An AT-Hook domain in MeCP2 determines the clinical course of Rett syndrome and related disorders

by Baker, S. A., Chen, L., Wilkins, A. D., Yu, P., Lichtarge, O., and Zoghbi, H. Y. (2013). Cell 152, 984-996.

Rett syndrome (RTT) is an X-linked neurodevelopmental disorder associated with intellectual disabilities, which almost exclusively affects females during early childhood with an incidence of 1:10,00015,000 worldwide (Neul and Zoghbi, 2004). RTT is primarily caused by lossof-function mutations in methyl-CpGbinding protein 2 (MECP2) (Amir et al., 1999), the gene encoding MeCP2, a transcriptional repressor that binds to methylated $\mathrm{CpG}$ sites in promoter regions of DNA (Lewis et al., 1992; Nan et al., 1997). But to date, effective treatments for RTT remain lacking, which makes the identification of critical MeCP2 function of great importance to decipher the molecular mechanisms of RTT pathogenesis. Recently, Baker et al. (2013) identified a highly conserved AT-hook domain important for $\mathrm{MeCP} 2$ function and closely related to clinical progressions observed in RTT.

$\mathrm{MeCP} 2$ is an abundant chromatinassociated nuclear protein with high affinity binding to DNA containing methyl-CpG throughout mammalian genomes (Lewis et al., 1992; Nan et al., 1997). MeCP2 contains two well-defined functional domains: an $\mathrm{N}$-terminal methyl-CpG binding domain (MBD) (Figure 1A), essential for its selective binding to 5-methylcytosine; and a central transcriptional repression domain
(TRD) (Figure 1A) that recruits the transcriptional co-repressor Sin3A, histone deacetylases (HDACs) (Jones et al., 1998; Nan et al., 1998) and other related chromatin-remodeling proteins (Chahrour and Zoghbi, 2007) (Figure 1B). In addition, a C-terminal domain (CTD) (Figure 1A) facilitates DNA binding and protein-protein interactions (Chandler et al., 1999; Buschdorf and Stratling, 2004). Sporadic mutations in MECP2 cause $95 \%$ cases of RTT, among which eight specific ones are the most common and account for $>65 \%$ of all individuals with RTT (R106W, R133C, T158M, R168X，R255X，R270X，R294X，R306C) (Figure 1A) (Calfa et al., 2011). Several phenotype-genotype correlation studies have contributed to gaining insight into the role of $\mathrm{MeCP} 2$ in brain development. In the February 28, 2013 issue of Cell, Baker and colleagues describe the generation of two new mouse lines bearing either a R270X or G273X truncation to mimic male RTT patients with R270fs and G273fs mutations, respectively. Characterizing these mice, they discovered that G273X mice exhibit a significantly delayed disease progression with a longer lifespan compared to R270X mice, which led to the hypothesis that the region between R270 and G273 is important for MeCP2 function.

How is that just 3 amino acids, have such significant consequences for the progression and severity of the mouse RTT-like phenotype? Using chromatin immunoprecipitation followed by sequencing (ChIP-seq), the authors found that both MeCP2-R270X and MeCP2G273X bind to DNA globally, very much the same as wildtype MeCP2 does. Then, they focused on the TRD (amino acids
207-310) where, these two mutations locate, and found that both mutations disrupt normal repressor activity of MeCP2. By looking more closely at gene expression over the course of disease, the authors discovered a delay in misregulation of a small subset of genes in G273X mice, although most of those genes eventually become misregulated as they do in R270X mice. Despite that MeCP2's repressor activity is disrupted in G273X mice, these mice lived longer and healthier than Mecp2 KO mice, which suggests additional functions.

A highly conserved AT-hook domain in MeCP2 that terminates at G273 (Figure 1A) answers the question. Adrian Bird's group had already described an AT-hook domain in MeCP2, but its function was unclear (Klose et al., 2005) (Figure 1A). AT-hooks are regions of a protein that bind to AT-rich DNA. $\mathrm{MeCP} 2$ requires an A/T-rich motif adjacent to methylated $\mathrm{CpG}$ dinucleotides for efficient DNA binding (Klose et al., 2005). Using an electrophoretic mobility shift assay (EMSA), the authors discovered a key difference between G273X and R270X mutants: loss of function of the second AT-hook domain impairs the DNA-binding ability of the R270X mutant, indicating that amino acids $270-272$ are essential for the DNA-binding feature of $\mathrm{MeCP} 2$. However, it is unclear if the probe used in the EMSA contained A/T-rich motives, which raises caution when considering this new MeCP2 feature as its most critical in transcriptional regulation. Prior work had shown the TRD-CTD domain of MeCP2 is largely responsible for facilitating oligomerization of nucleosomal arrays, as well as chromatin compaction (Ghosh et al., 2010). Using an in vitro assay of chromatin compaction, 


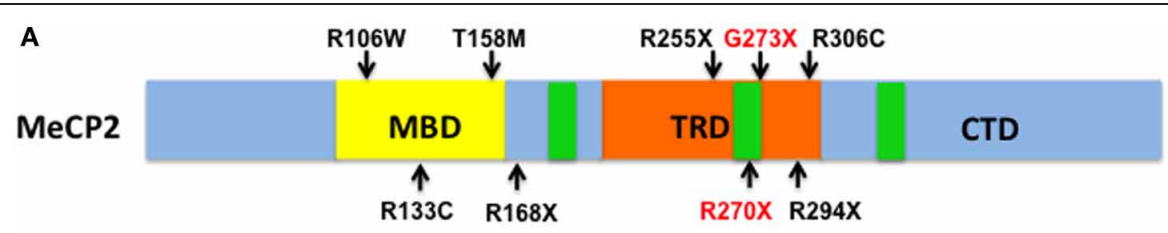

AT-hook domain

B

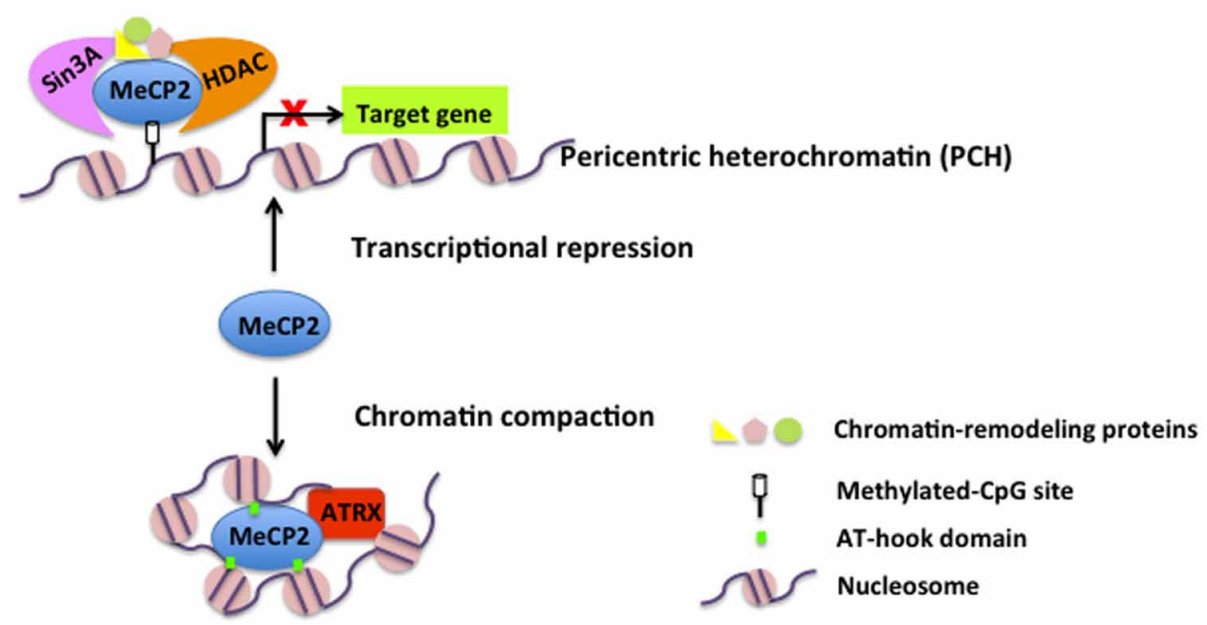

FIGURE 1 | MeCP2 structure and function in gene regulation and chromatin remodeling. (A) A schematic diagram of MeCP2 structure with functional domains and three AT-hook domains. Eight common MeCP2 mutations causing RTT and the two mutants discussed by Baker et al. (2013) are indicated in black and red, respectively. Note that R270X is also one of the most common mutations found in RTT individuals. (B) The function of
MeCP2 in transcription repression and chromatin compaction. As a transcriptional repressor, MeCP2 recruits Sin3A, histone deacetylases (HDACs), and some chromatin-remodeling proteins to silence target gene transcription. Also, Baker et al. (2013) discovered that AT-hook clusters facilitate MeCP2 to alter chromatin conformation by recruitment of ATRX to $\mathrm{PCH}$ to maintain chromatin homeostasis.
Baker et al. (2013) show that MeCP2R270X does not compact chromatin as efficiently as MeCP2-G273X does. These results not only provide clear evidence that this second AT-hook domain plays an important role in manipulating chromatin structure, but also explain the phenotypic differences observed in G273X and R270X mice. The authors propose that, after the MBD of MeCP2 binds to methylated DNA regions, the AT-hook clusters participate in altering DNA conformation to aid in chromatin packing (Figure 1B).

Furthermore, Baker and colleagues showed that the localization of $\alpha$-thalassemia/mental retardation syndrome X-linked protein (ATRX) to pericentric heterochromatin $(\mathrm{PCH})$ is a distinguishing feature between G273X and R270X. This is an intriguing finding not only because ATRX syndrome shows overlap with RTT, but also because ATRX participates in chromatin remodeling, associates with $\mathrm{MeCP} 2$, and disruption of that interaction is thought to contribute to intellectual disability (Nan et al., 2007). The new findings by Baker et al. (2013) strongly suggest that ATRX contributes to the function of MeCP2 on chromatin modification (Figure 1B). It would be interesting to further explore the role of ATRX in MeCP2 dysfunction not only in RTT individuals, but also in other intellectual disabilities associated with MECP2 mutations.

In summary, Baker et al. (2013) identified a novel AT-hook domain of MeCP2 that plays an important role in chromatin organization, providing a new model involving an additional protein partner, which, incidentally, is also implicated in a neurodevelopmental disorder associated with intellectual disability. They took advantage of the correlation between mouse models and human individuals, identified the pathogenesis of various RTT-like phenotypes in novel mouse lines, which increased our understanding of how different MeCP2 functions are affected by various disease-causing mutations. In this regard, we very much share the hopes of the authors that their discovery of a new fundamental feature of MeCP2 can further help in developing novel therapeutic approaches for RTT and other intellectual disabilities associated with MECP2 mutations.

\section{REFERENCES}

Amir, R. E., Van den Veyver, I. B., Wan, M., Tran, C. Q., Francke, U., and Zoghbi, H. Y. (1999). Rett syndrome is caused by mutations in X-linked MECP2, encoding methyl-CpG-binding protein 2. Nat. Genet. 23, 185-188.

Baker, S. A., Chen, L., Wilkins, A. D., Yu, P., Lichtarge, O., and Zoghbi, H. Y. (2013). An AT-Hook domain in MeCP2 determines the clinical course of Rett syndrome and related disorders. Cell 152, 984-996.

Buschdorf, J. P., and Stratling, W. H. (2004). A WW domain binding region in methyl-CpG-binding protein MeCP2: impact on Rett syndrome. J. Mol. Med. 82, 135-143.

Calfa, G., Percy A. K., and Pozzo-Miller, L. (2011). Experimental models of Rett syndrome based on Mecp2 dysfunction. Exp. Biol. Med. 236, 3-19. 
Chahrour, M., and Zoghbi, H. Y. (2007). The story of Rett syndrome: from clinic to neurobiology. Neuron 56, 422-437.

Chandler, S. P., Guschin, D., Landsberger, N., and Wolffe, A. P. (1999). The methyl-CpG binding transcriptional repressor $\mathrm{MeCP} 2$ stably associates with nucleosomal DNA. Biochemistry 38, 7008-7018.

Ghosh, R. P., Nikitina, T., Horowitz-Scherer, R. A., Gierasch, L. M., Uversky, V. N., Hite, K., et al. (2010). Unique physical properties and interactions of the domains of methylated DNA binding protein 2. Biochemistry 49, 4395-4410.

Jones, P. L., Veenstra, G. J., Wade, P. A., Vermaak, D., Kass, S. U., Landsberger, N., et al. (1998). Methylated DNA and $\mathrm{MeCP} 2$ recruit histone deacetylase to repress transcription. Nat. Genet. 19, 187-191.

Klose, R. J., Sarraf, S. A., Schmiedeberg, L., McDermott, S. M., Stancheva, I., and Bird,
A. P. (2005). DNA binding selectivity of MeCP2 due to a requirement for $\mathrm{A} / \mathrm{T}$ sequences adjacent to methyl-CpG. Mol. Cell 19, 667-678.

Lewis, J. D., Meehan, R. R., Henzel, W. J., MaurerFogy, I., Jeppesen, P., Klein, F., et al. (1992). Purification, sequence, and cellular localization of a novel chromosomal protein that binds to methylated DNA. Cell 69, 905-914.

Nan, X., CamPoy, F. J., and Bird, A. (1997). $\mathrm{MeCP} 2$ is a transcriptional repressor with abundant binding sites in genomic chromatin. Cell 88 , 471-481.

Nan, X., Hou, J., Maclean, A., Nasir, J., Lafuente, M. J., Shu, X., et al. (2007). Interaction between chromatin proteins MECP2 and ATRX is disrupted by mutations that cause inherited mental retardation. Proc. Natl. Acad. Sci. U.S.A. 104, 2709-2714.

Nan, X., Ng, H. H., Johnson, C. A., Laherty, C. D., Turner, B. M., Eisenman, R. N., et al. (1998).
Transcriptional repression by the methyl-CpGbinding protein MeCP2 involves a histone deacetylase complex. Nature 393, 386-389.

Neul, J. L., and Zoghbi, H. Y. (2004). Rett syndrome: a prototypical neurodevelopmental disorder. Neuroscientist 10, 118-128.

Received: 01 April 2013; accepted: 18 April 2013; published online: 09 May 2013.

Citation: Xu X and Pozzo-Miller L (2013) A novel DNA-binding feature of MeCP2 contributes to Rett syndrome. Front. Cell. Neurosci. 7:64. doi: 10.3389/fncel. 2013.00064

Copyright (C) 2013 Xu and Pozzo-Miller. This is an open-access article distributed under the terms of the Creative Commons Attribution License, which permits use, distribution and reproduction in other forums, provided the original authors and source are credited and subject to any copyright notices concerning any thirdparty graphics etc. 